\title{
The challenge of defining exacerbation in bronchiectasis
}

\author{
Miguel Ángel Martínez-García ${ }^{1}$ and Luis Máiz-Carro²
}

\begin{abstract}
Affiliations: ${ }^{1}$ Pneumology Department, Hospital Universitario y Politécnico La Fe de Valencia, Valencia, Spain. ${ }^{2}$ Bronchiectasis and Cystic Fibrosis Unit, Pneumology Department, Hospital Universitario Ramón y Cajal, Madrid, Spain.
\end{abstract}

Correspondence: Miguel Ángel Martínez-García, Pneumology Department, Hospital Universitario y Politécnico La Fe de Valencia, Avenida Fernando Abril Martorell 106, 46026 Valencia, Spain. E-mail: mianmartinezgarciaA gmail.com

@ERSpublications

A worldwide consensus has been reached in the definition of exacerbation in bronchiectasis for research purposes http://ow.ly/SD2P30bFBjX

Cite this article as: Martínez-García MÁ, Máiz-Carro L. The challenge of defining exacerbation in bronchiectasis. Eur Respir J 2017; 49: 1700700 [https://doi.org/10.1183/13993003.00700-2017].

One of the most difficult aspects of the study of chronic respiratory diseases is the concept of exacerbation. Defining the limits of each patient's normal daily variations in symptoms, and the minimum time beyond these limits that can be considered the start of an acute process of the disease, poses a challenge that is often insuperable. It is also just as difficult to establish the specific parameters that must be used to define an exacerbation; parameters that are usually grouped as symptoms and/or factors involving the use of health resources or a need for treatment. Nevertheless, in the case of bronchiectasis, and other respiratory diseases, exacerbations (particularly severe exacerbations) have a demonstrable negative impact on a patient's prognosis [1-4] and entail great expense [5, 6], and so their identification, and therefore their definition, is crucial to any early detection and treatment. Ideally, such a definition should present a perfect diagnostic value and, as regards bronchiectasis, be based on reproducible objective measurements linked to inflammation/bronchial infection (biomarkers) and their consequences (acute clinical symptoms). At the moment, however, this approach is not viable for various reasons: we do not have any available biomarkers with sufficient diagnostic value, although there have been some promising studies in this respect [7]; we do not have any sufficiently specific objective measurements for the key symptoms in exacerbations; and, finally, we need to know a patient's previous inflammatory/infectious state in order to quantify the change caused by the supposed exacerbation. Furthermore, there is a significant problem here as a review of the literature reveals a wide range of definitions of bronchiectasis, depending on the studies analysed and the mixture of objective and subjective criteria, making it impossible to compare their results.

There is no reason to despair, however, as there is another hugely important factor that is already within our reach and would undoubtedly mark a big step forward: the opportunity to establish the broadest possible consensus on the definition of exacerbation that should be adopted in both the clinical and the scientific fields, even if we have to assume a certain degree of error because of uncontrollable intra- and interindividual variability. Such a consensus would enhance our capacity to compare the results of different studies, thus furthering our knowledge of exacerbations of bronchiectasis and their treatment.

In the current issue of the European Respiratory Journal HiLl et al. [8] publish a study in which they succeed in doing precisely that: they establish a consensus by gathering a broad-based group of experts in bronchiectasis from all over the world to make an operative definition of an exacerbation of bronchiectasis

Received: April 032017 | Accepted: April 042017

Conflict of interest: None declared.

Copyright OERS 2017 
that can be applied to the scientific field. This definition is based on the appearance of at least three out of six symptoms that require a change in treatment, related to coughing, sputum characteristics, dyspnoea and tiredness for at least $48 \mathrm{~h}$. Although it is very possible that all the experts on the panel, and other researchers who did not take part, could present examples of patients with apparent exacerbations not covered by this definition (for example, increased coughing and sputum purulence developing over $48 \mathrm{~h}$ that require antibiotic treatment, or various clinical criteria fulfilled for less than $48 \mathrm{~h}$ that require early antibiotic treatment), the very fact that they reached an agreement is ample reason to congratulate these authors. One important point, fully explained by the authors, is that this is a definition for use in research, as it may have a high specificity (little probability of identifying exacerbations that are not in fact exacerbations). This leads to selection bias towards more severe exacerbations, although in a research context it is preferable to err in this direction as it is the severe forms that most seem to affect a patient's prognosis [1-4], and they are thus the most appropriate target or inclusion criterion for clinical studies. However, the definition does incorporate a number of symptomatic elements, and the need to change treatment (in this case, to prescribe antibiotics), which may contribute towards a better balance between sensitivity and specificity. It is also important to make the distinction between this definition, intended for research purposes, and one applicable to clinical care. The latter would maybe require an even broader consensus and should also have greater sensitivity to avoid any nontreatment of an exacerbation because of a failure to identify it. Finally, the definition proposed by the authors of this study needs an external validation in other areas such as Asia, where there could be variations in the perception of symptoms because of the different cultural situation, as well as different settings and degrees of access to treatment.

In a globalised world with increased ease of communication, it does not seem right that regulations, guidelines, consensuses, etc., establish different parameters to define an exacerbation of bronchiectasis (or other circumstances). It hardly seems reasonable that, depending on the geographical area in which patients find themselves, the very same disease or exacerbation can be defined differently and therefore treated differently. It is therefore appropriate to congratulate the drive towards a consensus on the part of the authors of this study and encourage them to broaden it to embrace many other researchers from various parts of the world and many other definitions that remain unclear with respect to bronchiectasis: the radiological and clinical criteria for diagnosis [9]; the concepts of colonisation and chronic/initial bronchial infection; bacterial eradication $[10,11]$ and phenotypical complexity [12-14].

\section{References}

1 Martínez-García MA, Soler-Cataluña JJ, Perpiñá-Tordera M, et al. Factors associated with lung function decline in adult patients with stable non-cystic fibrosis bronchiectasis. Chest 2007; 132: 1565-1572.

2 Martínez-García MA, Athanazio R, Girón R, et al. Predicting high risk of exacerbations in bronchiectasis: the E-FACED score. Int J COPD 2017; 12: 275-284.

3 Rosales-Mayor E, Polverino E, Raguer L, et al. Comparison of two prognostic (BSI and FACED) in a Spanish cohort of adult patients with bronchiectasis and improvement of the FACED predictive capacity for exacerbations. PloS ONE 2017; 12: e0175171.

4 Chalmers JD, Goeminne P, Aliberti S, et al. The Bronchiectasis Severity Index. An international derivation and validation study. Am J Respir Crit Care Med 2014; 189: 576-585.

5 De la Rosa D, Martínez-García MA, Olveira C, et al. Annual direct medical costs of bronchiectasis treatment: impact of severity, exacerbations, chronic bronchial colonization and COPD coexistence. Chron Respir Dis 2016; in press [https://doi.org/10.1177/1479972316643698].

6 Seitz AE, Olivier KN, Steiner CA, et al. Trends and burden of bronchiectasis-associated hospitalizations in the United States, 1993-2006. Chest 2010; 138: 944-949.

7 Chalmers JD, Moffitt KL, Suarez-Cuartin G, et al. Neutrophil elastase activity is associated with exacerbations and lung function decline in bronchiectasis. Am J Respir Crit Care Med 2016; 195: 1384-1393.

8 Hill AT, Haworth CS, Aliberti S, et al. Pulmonary exacerbation in adults with bronchiectasis: a consensus definition for clinical research. Eur Respir J 2017; 49: 1700051.

9 Naidich DP, McCauley DI, Khouri NF, et al. Computed tomography of bronchiectasis. J Comput Assist Tomogr 1982; 6: 437-444.

10 Vendrell M, de Gracia J, Olveira C, et al. Spanish Society of Pneumology and Thoracic Surgery. Diagnosis and treatment of bronchiectasis [in Spanish]. Arch Bronconeumol 2008; 44: 629-640.

11 Pasteur MC, Bilton D, Hill AT, et al. British Thoracic Society guideline for non-CF bronchiectasis. Thorax 2010; 65: Suppl. 1, il-i58.

12 Martínez-García MA, Vendrell M, Girón R, et al. The multiple faces of non-cystic fibrosis bronchiectasis. A cluster analysis approach. Ann Am Thorac Soc 2016; 13: 1468-1475.

13 Aliberti S, Lonni S, Dore S, et al. Clinical phenotypes in adult patients with bronchiectasis. Eur Respir J 2016; 47: 1113-1122.

14 Chalmers JD. Bronchiectasis: phenotyping a complex disease. COPD 2017; 14: Suppl. 1, S12-S18. 